\title{
Exact solutions of embedding the 4D Universe in a 5D Einstein manifold
}

\author{
Xin-He Meng $1,3,4$ Jie Ren 2 i and Hong-Guang Zhang ${ }^{1}$ \\ ${ }^{1}$ Department of physics, Nankai University, Tianjin 300071, China \\ ${ }^{2}$ Theoretical Physics Division, Chern Institute of Mathematics, Nankai University, Tianjin 300071, China \\ ${ }^{3}$ BK21 Division of Advanced Research and Education in physics, Hanyang University, Seoul 133-791, Korea and \\ ${ }^{4}$ Department of physics, Hanyang University, Seoul 133-791, Korea
}

(Dated: October 25, 2018)

\begin{abstract}
One of the simplest way to extend 4D cosmological models is to add another spatial dimension to make them 5D. In particular, it has been shown that the simplest of such 5D models, i.e., one in which the right hand side of the Einstein equation is empty, induces a 4D nonempty Universe. Accordingly, the origin of matter in the 4D real Universe might be mathematically attributed to the existence of one (fictitious) extra spatial dimension. Here we consider the case of an empty 5D Universe possessing a cosmological constant $\Lambda$ and obtain exact solutions for both positive and negative values of $\Lambda$. It is seen that such a model can naturally reduce to a power law $\Lambda C D M$ model for the real Universe. Further, it can be seen that, the arbitrary constants and functions appearing in this model are endowed with definite physical meanings.
\end{abstract}

PACS numbers: 04.50.+h,98.80.-k

\section{INTRODUCTION}

It is very likely that the expansion of our Universe is currently in an accelerating phase, supported by the most direct evidence from the measurements of type Ia supernova, and others such as the observations of CMB by the WMAP satellite, large-scale galaxy surveys by $2 \mathrm{dF}$ and SDSS [1, 2, 3]. But now the mechanisms responsible for the accelerating expansion are not very clear. Many authors introduce a mysterious cosmic fluid called dark energy to explain this (see Ref. [4] for a review). On the other hand, some authors suggest that maybe there does not exist such mysterious dark energy, but instead the observed cosmic acceleration is a signal of our first real lack of understanding of gravitational physics [5]. An example is the braneworld theory with the extra dimensions compactified or noncompactified [6, 7, 8]. Many cosmological models are inspired from the string theory, which may be incorrect at the worst and speculative at the best [9]. Despite the apparent evidence for an accelerated expansion, there is also some "neglected" but important experimental evidence against the $\Lambda$ Cold Dark Matter $(\Lambda \mathrm{CDM})$ model, which is the mainstream model in cosmology [10]. Nevertheless, given the presently popular notion, it is reasonable to study the cosmological models that can be reduced to the $\Lambda \mathrm{CDM}$ model, as in our previous works [11] and the present one.

Space-Time-Matter (STM) theory [12, 13, 14, 15] suggests that our Universe is a four-dimensional (4D) hyperspace embedded in a 5D Ricci-flat manifold, i.e., the matter in the 4D real Universe is induced by a 5D Ricci-flat Universe. Leon has shown that the braneworld theory and the induced-matter theory are actually equivalent [16]. Solutions of the 5D Einstein equation without any matter source can always be regarded as solutions of the corresponding $4 \mathrm{D}$ gravitational field equations with the induced matter, as the Compbell theorem indi-

\footnotetext{
*Electronic address: xhm@ nankai.edu.cn

${ }^{\dagger}$ Electronic address: jrenphysics@hotmail.com
}

cates [17]. However, in the previous work the 5D Universe is assumed to be Ricci flat, i.e., without a 5D cosmological constant. In the present work, we assume that a cosmological constant exists in the 5D Universe as a more general case to study the features of the model, and demonstrate that the reduction to the 4D real Universe can be realized by a simple choice of the arbitrary functions in the metric. At the same time, each function and constant in the metric separately possesses explicit physical meanings.

The paper is planned as follows. In the next section we review how a solution of 5D Ricci-flat Universe in the inducedmatter theory can be used to generate a simple 4D cosmology. In section 3 we give the exact solutions of the 5D Universe with a cosmological constant and study the new features. The last section devotes discussions and conclusions for the general framework studies to this toy model.

\section{5D RICCI-FLAT UNIVERSE}

Firstly, we summarize some basics of STM theory [12, 13, 14, 15], as a preparation for our work. STM theory starts with a solution of Einstein's equation in 5D Ricci-flat spacetime,

$$
R_{a b}=0,
$$

where the subscript indices run from 0 to 4 . A new class of solution for Eq. (1), which extends the FRW solutions, is given in Ref. [14] (and we take their conventions hereafter, i.e., we work in natural units where $c=8 \pi G=1$ ):

$$
\begin{aligned}
d s^{2} & =B^{2} d t^{2}-A^{2}\left(\frac{d r^{2}}{1-k r^{2}}+r^{2} d \Omega^{2}\right)-d y^{2}, \\
A^{2}(t, y) & =\left(\mu^{2}+k\right) y^{2}+2 v y+\frac{v^{2}+K}{\mu^{2}+k}, \\
B(t, y) & =\frac{1}{\mu} \frac{\partial A}{\partial t} \equiv \frac{\dot{A}}{\mu} .
\end{aligned}
$$

Here $\mu=\mu(t)$ and $v=v(t)$ are arbitrary functions, $k$ is the 3D curvature index $(k= \pm 1,0)$ and $K$ is a constant that relates the 
5D Kretschmann invariant as the form $R_{a b c d} R^{a b c d}=72 K^{2} / A^{8}$, which shows that $K$ determines the curvature of the 5D manifold. This framework has been intensely studied in the literature [15, 18, 19]. The spacetime geometry is encoded in the functions $A$ and $B$, which are dependent on the extra dimension $y$. Since the form of $B d t$ is invariant under an arbitrary transformation $t=t(\widetilde{t})$, one of the two arbitrary functions $\mu(t)$ and $v(t)$ can be fixed without changing the generality of the solution. Here $\mu(t)=0$ corresponds to a singularity, and if a $\mathbb{Z}_{2}$ symmetry of the metric with respect to the extra dimension $y$ is required, the another free function $v(t)$ must disappear.

The corresponding $4 \mathrm{D}$ line element is

$$
d s_{4}^{2}=g_{\alpha \beta} d x^{\alpha} d x^{\beta}=B^{2} d t^{2}-A^{2}\left(\frac{d r^{2}}{1-k r^{2}}+r^{2} d \Omega^{2}\right),
$$

which is similar to the Robertson-Walker metric and thus underlies the standard FRW models. We can calculate the nonvanishing components of the 4D Ricci tensor and its corresponding scalar. The $4 \mathrm{D}$ Einstein tensor ${ }^{(4)} G_{\beta}^{\alpha} \equiv{ }^{(4)} R_{\beta}^{\alpha}-$ $\delta_{\beta}^{\alpha(4)} R / 2$ with its non-vanishing components is

$$
\begin{aligned}
& { }^{(4)} G_{0}^{0}=\frac{3\left(\mu^{2}+k\right)}{A^{2}}, \\
& { }^{(4)} G_{1}^{1}={ }^{(4)} G_{2}^{2}={ }^{(4)} G_{3}^{3}=\frac{2 \mu \dot{\mu}}{A \dot{A}}+\frac{\mu^{2}+k}{A^{2}} .
\end{aligned}
$$

These give the components of the induced energy-momentum tensor since Einstein's equation $G_{\beta}^{\alpha}=T_{\beta}^{\alpha}$ holds. Provided that the induced matter is described by a perfect fluid with density $\rho$ and pressure $p$ moving with a 4-velocity $u^{\alpha} \equiv d x^{\alpha} / d s$, plus a cosmological term whose nature is to be determined, the energy-momentum tensor is given by

$$
{ }^{(4)} G_{\alpha \beta}=(\rho+p) u_{\alpha} u_{\beta}+\left(\rho_{\Lambda}-p\right) g_{\alpha \beta} .
$$

The above framework provides special cases with confrontations to physics observations. For a braneworld model, the authors of Ref. [20] have studied a solution that incorporates the $\mathbb{Z}_{2}$ reflection symmetry condition $g_{\alpha \beta}\left(x^{\gamma}, y\right)=$ $g_{\alpha \beta}\left(x^{\gamma},-y\right)$, which can be achieved directly by setting $v=0$ in the metric solution. Different choices and the corresponding forms of $A(t, y)$ and $B(t, y)$ may give differently concrete models, which can be found in Refs. [12, 15, 18, 19]. At present we are most interested in the evolution of the Universe including the so called "dark energy" (if it really exists without the possible new signature that our gravity is to be modified at cosmic scale).

\section{REDUCTION TO $\Lambda$ CDM MODEL IN THE 5D UNIVERSE WITH COSMOLOGICAL CONSTANT}

We assume that a cosmological constant $\Lambda$ exists in the 5D Universe as a more general case. Starting with the ansatz of Eq. (2), we solve Einstein's equation $R_{a b}-\frac{1}{2} g_{a b} R=\Lambda g_{a b}$ and obtain the solution for $\Lambda>0$,

$$
\begin{aligned}
& A(t, y)^{2}=\frac{2\left(\mu^{2}+k\right)(1-\cos \lambda y)}{\lambda^{2}}+\frac{2 v \sin \lambda y}{\lambda} \\
+ & \frac{2\left[\mu^{2}+k-\sqrt{\left(\mu^{2}+k\right)^{2}-\lambda^{2}\left(v^{2}+K\right)}\right] \cos \lambda y}{\lambda^{2}},
\end{aligned}
$$

where $\lambda=\sqrt{6 \Lambda} / 3, \mu=\mu(t)$ and $v=v(t)$. And $B(t, y)=\dot{A} / \mu$ remains unchanged. For the case $\Lambda<0$, by defining $\lambda=$ $\sqrt{6|\Lambda|} / 3$, the solution is

$$
\begin{array}{r}
A(t, y)^{2}=\frac{2\left(\mu^{2}+k\right)(\cosh \lambda y-1)}{\lambda^{2}}+\frac{2 v \sinh \lambda y}{\lambda} \\
+\frac{2\left[\sqrt{\left(\mu^{2}+k\right)^{2}+\lambda^{2}\left(v^{2}+K\right)}-\left(\mu^{2}+k\right)\right] \cosh \lambda y}{\lambda^{2}},
\end{array}
$$

These solutions can be reduced to Eq. (3) by taking the limit $\lambda \rightarrow 0$. We will show that the positive $\Lambda$ induces a positive cosmological constant in the 4D Universe under some conditions. For simplicity we first consider the case $k=K=y=0$; then

$$
A^{2}=\frac{2\left(\mu^{2}-\sqrt{\mu^{2}+\lambda^{2} v^{2}}\right)}{\lambda^{2}} .
$$

Now we substitute the simple choice of $\mu(t)$ and $v(t)$

$$
\mu(t)=\dot{A}, \quad v(t)=\sqrt{C A},
$$

where $C$ is a parameter, to Eq. (11). After some arrangements, this solution can be naturally reduced to the form

$$
\begin{aligned}
H^{2} & =C A^{-3}+\frac{\lambda^{2}}{4} \\
& =H_{0}^{2}\left[\Omega_{m} A^{-3}+\Omega_{\Lambda}\right],
\end{aligned}
$$

where $H=\dot{A} / A, \Omega_{m}=C / H_{0}^{2}$, and $\Omega_{\Lambda}=\Lambda /\left(6 H_{0}^{2}\right)$. This is essentially the $\Lambda \mathrm{CDM}$ model, and the cosmological constant in the 5D Universe contributes a term as the cosmological constant in the 4D real Universe.

In the general case, taking $y=0$ in Eq. (9) gives

$$
A^{2}=\frac{2\left[\mu^{2}+k-\sqrt{\left(\mu^{2}+k\right)^{2}-\lambda^{2}\left(v^{2}+K\right)}\right]}{\lambda^{2}} .
$$

By substituting Eq. (12) in Eq. (14), we obtain

$$
\begin{aligned}
H^{2} & =C A^{-3}-k A^{-2}+K A^{-4}+\frac{\lambda^{2}}{4} \\
& =H_{0}^{2}\left[\Omega_{m} A^{-3}-\Omega_{k} A^{-2}+\Omega_{K} A^{-4}+\Omega_{\Lambda}\right],
\end{aligned}
$$

where $\Omega_{k}=k / H_{0}^{2}$ and $\Omega_{K}=K / H_{0}^{2}$. It turns out that the constant $K$ contributes a term that describes radiation. Conventionally, the redshift is defined by $z=1 / A-1$, thus $A^{-1}=1+z$. Compared with the expression of $H^{2}$ for the power law $\Lambda C D M$ model, we can see that from this 5D Universe with cosmological constant, the arbitrary functions and constants in the metric are endowed with explicit physical meanings,

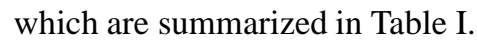


TABLE I: Physical meanings of functions and constants in the metric of the 5D Universe

\begin{tabular}{ccc}
\hline \hline Functions or constants & Physical meanings & Terms in $H^{2}$ \\
\hline$\mu(t)=\dot{A}$ & Velocity & \\
$v(t)=\sqrt{C A}$ & Matter (dust) & $\Omega_{m}(1+z)^{3}$ \\
$k$ & Curvature & $\Omega_{k}(1+z)^{2}$ \\
$K$ & Radiation & $\Omega_{K}(1+z)^{4}$ \\
$\Lambda$ & Cosmological constant & $\Omega_{\Lambda}$ \\
\hline \hline
\end{tabular}

The reduction to the $\Lambda C D M$ model can be also realized in 5D Ricci-flat Universe by the choice

$$
\mu(t)=\dot{A}, \quad v(t)=\tilde{H}(z) A^{2},
$$

where $\tilde{H}(z)$ describes the $\Lambda \mathrm{CDM}$ model as

$$
\tilde{H}(z)^{2}=H_{0}^{2}\left[\Omega_{m}(1+z)^{3}+1-\Omega_{m}\right] .
$$

Thus, the general case with $y=0$ gives

$$
H(z)^{2}=\tilde{H}(z)^{2}-k(1+z)^{2}+K(1+z)^{4} .
$$

Of course we can encode more physics in the function $v(t)$, but in the 5D Universe with cosmological constant, each arbitrary function and constant separately possesses physical meanings, which is more elegant.

\section{CONCLUSION}

We have presented the exact solutions of embedding the 4D Universe in a 5D Einstein manifold, and investigated the 5D cosmological model with cosmological constant and the reduction to the power law $\Lambda \mathrm{CDM}$ model for the 4D real Universe. As a generalization of the $5 \mathrm{D}$ Ricci-flat solution in STM theory, the 5D solution with cosmological constant has new phenomenological features. Each function and constant in the metric can separately possess explicit physical meanings when we perform a $4 \mathrm{D}$ reduction. We can also study the general properties of the induced matter as the contents in our Universe, without specifying the form of the arbitrary functions. The phantom case can also be realized, for example, the equation of state parameter $w=p / \rho<-1$, if we take $\lambda=k=v=0$ and $K=1$. We think this picture is in conformity with other models to explain the late-time accelerating expansion of our Universe, thus it is worth of further endeavors.

\section{Acknowledgements}

J.R. thanks Prof. Liu Zhao for helpful discussions. X.-H.M. is supported partly by NSFC under No. 10675062 and partly by the 2nd stage Brain Korea 21 Program.
[1] S. Perlmutter el al. Nature 404 (2000) 955; Astroph. J. 517 (1999) 565; A. Riess et al. Astroph. J. 116 (1998) 1009; astro-ph/0611572

[2] D.N. Spergel, et al., ApJS, 170 (2007) 377.

[3] B. Roukema, et al., Astron. Astrophys. 382 (2002) 397; D.J. Eisenstein et al., Astrophys. J. 633, (2005) 560.

[4] P.J.E. Peebles and B. Ratra, Rev. Mod. Phys. 75 (2003) 559; S.M. Carroll, Living Rev. Rel. 4 (2001) 1; T. Padmanabhan, Phys. Rep. 380 (2003) 235.

[5] A. Lue, R. Scoccimarro, and G. Starkman, Phys. Rev. D 69 (2004) 044005; X.H. Meng and P. Wang, Class. Quant. Grav. 20 (2003) 4949; 21 (2004) 951; 21 (2004) 2029; 22 (2005) 23; Phys. Lett. B 584 (2004) 1, for example.

[6] G. Dvali, G. Gabadadze and M. Porrati, Phys. Lett. B 485 (2000) 208.

[7] L. Randall, R. Sundrum, Phys. Rev. Lett. 83 (1999) 3370; 83 (1999) 4690.

[8] P. Hořava, E. Witten, Nucl. Phys. B. 460 (1996) 506; 475 (1996) 94.

[9] P. Woit, Not Even Wrong: The Failure of String Theory And the Search for Unity in Physical Law (Basic Books, New York, 2006).

[10] R. Lieu, arXiv:0705.2462 [astro-ph].

[11] J. Ren and X.H. Meng, Phys. Lett. B 633 (2006) 1; 636 (2006) 5; Phys. Rev. D 76 (2007) 043521.
[12] P.S. Wesson, Gen. Rel. Grav. 16 (1984) 193; P.S. Wesson, Space-Time-Matter, (World Scientific, Singapore, 1999); P.S. Wesson, Gen. Rel. Grav. 19 (2002) 2825; P.S. Wesson, Found. Phys. Lett. 19 (2006) 285, and refereneces therein.

[13] P.S. Wesson, J. Ponce de Leon, J. Math. Phys. 33 (1992) 3883.

[14] H.Y. Liu and B. Mashhoon, Ann. Phys. 4 (1995) 565.

[15] H.Y. Liu and P.S. Wesson, Astrophys. J. 562 (2001) 1.

[16] J. Ponce de Leon, Mod. Phys. Lett. A. 16 (2001) 2291.

[17] J.E. Campbell, A Course of Differential Geometry (Clarendon, Oxford, 1926); S.S. Seahra and P.S. Wesson, Class. Quant. Grav. 20 (2003) 1321.

[18] C.W. Zhang, H.Y. Liu, L.X. Xu, and P.S. Wesson, Mod. Phys. Lett. A 21 (2006) 571.

[19] L.X. Xu, H.Y. Liu, and B.L. Wang, Chin. Phys. Lett. 20 (2003) 995, gr-qc/0304049, L.X. Xu and H.Y. Liu, Int. J. Mod. Phys. D 14 (2005) 883, astro-ph/0412241; L.X. Xu, H.Y. Liu, and B.R. Chang, Mod. Phys. Lett. A 20 (2005) 3105, astro-ph/0507397; L.X. Xu, H.Y. Liu, and C.W. Zhang, Int. J. Mod. Phys. D 15 (2006) 215, astro-ph/0510673, L.X. Xu, H.Y. Liu, and Y.L. Ping, Int. J. Theor. Phys. 45 (2006) 843, astro-ph/0601471; Y.L. Ping, H.Y. Liu, and L.X. Xu, Int. J. Mod. Phys. A 22 (2007) 985, gr-qc/0610094.

[20] T. Liko and P.S. Wesson, Int. J. Mod. Phys. A 20 (2005) 2037, gr-qc/0310067. 